\title{
Propulsion Deceleration Studies using Planar Laser-Induced Iodine Fluorescence and Computational Fluid Dynamics
}

\author{
James C. McDaniel, ${ }^{1}$ Joshua R. Codoni, ${ }^{2}$ Erin M. Reed ${ }^{3}$ \\ Department of Mechanical and Aerospace Engineering, University of Virginia, Charlottesville, VA, 22903 \\ Hicham Alkandry ${ }^{4}$ and Iain D. Boyd ${ }^{5}$ \\ Department of Aerospace Engineering, University of Michigan, Ann Arbor, MI, 48109
}

Future high-mass spacecraft entering the thin Martian atmosphere will require additional means of deceleration prior to deploying supersonic parachutes. Propulsive deceleration is one technology that is being considered. The interaction of the spacecraft aerodynamics with the propulsion deceleration (PD) jets has been shown to cause a decrease in drag coefficient with increasing thrust coefficient, which is not desirable for deceleration. Planar LaserInduced Iodine Fluorescence (PLIIF) images showed a lifting of the vehicle bow shock away from the aeroshell. Flowfield calculations performed using a CFD code showed that this lifting was responsible for the decrease in drag with increasing PD jet thrust. With 4 PD jets located midway between the aeroshell centerline and shoulder, PLIIF images showed that the vehicle bow shock is maintained between the jets as the thrust coefficient is increased. CFD calculations established that this bow shock was responsible for greater drag preservation with the peripheral jets. The peripheral jet drag coefficient was 4 times larger than the single jet value at a thrust coefficient of 2.0. The calculations also showed low pressure wakes located radially behind the peripheral jets which are responsible for the decrease in drag coefficient with increasing thrust coefficient and that high pressure is maintained between the jets. These results suggest that using a few peripheral PD jets located near the aeroshell shoulder would provide the greatest amount of drag preservation when using propulsive deceleration.

\section{Nomenclature}

$\begin{array}{ll}C_{D} & =\text { Coefficient of Drag } \\ C_{T} & =\text { Thrust Coefficient } \\ D & =\text { Aeroshell Diameter }[\mathrm{m}] \\ M & =\text { Mach Number } \\ \dot{m} & =\text { Mass Flow Rate }[\mathrm{kg} / \mathrm{s}] \\ p & =\text { Pressure }\left[\mathrm{N} / \mathrm{m}^{2}\right] \\ q & =\text { Dynamic Pressure }\left[\mathrm{N} / \mathrm{m}^{2}\right] \\ \mathrm{S} & =\text { Aeroshell Frontal Area }\left[\mathrm{m}^{2}\right] \\ \mathrm{S}_{\mathrm{F}} & =\text { Fluorescence Signal } \\ \mathrm{T} & =\text { Temperature } \\ T & =\text { Thrust }[\mathrm{N}] \\ \mathrm{u} & =\text { Velocity }[\mathrm{m} / \mathrm{s}] \\ \theta_{\text {rot }} & =\text { Characteristic Rotational Temperature }[\mathrm{K}] \\ \theta_{\text {vib }} & =\text { Characteristic Vibrational Temperature }[\mathrm{K}] \\ \chi & =\text { Mole Fraction }\end{array}$

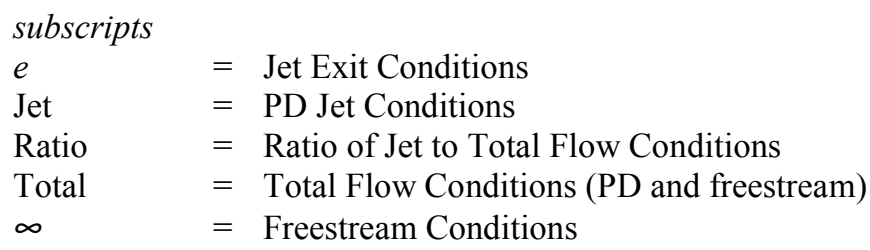

\footnotetext{
${ }^{1}$ Professor, Associate Fellow AIAA.

${ }^{2}$ Graduate Student, Student Member AIAA.

${ }^{3}$ Graduate Student, Student Member AIAA.

${ }^{4}$ Graduate Student, Student Member AIAA.

${ }^{5}$ Professor, Fellow AIAA.
} 


\section{Introduction}

$\mathrm{F}$ UTURE human-scale missions to Mars will have landed masses of about two orders of magnitude greater than previous robotic missions. This makes the challenge of entry, descent and landing extremely difficult owing to the fact that the Martian atmosphere has a density about 1\% that on Earth. Missions to date, even including the Mars Science Laboratory to launch this year, have used technology dating from the Viking era, consisting of blunt 70 degree sphere cones and supersonic disk-gap-band parachutes. Deceleration to parachute deployment speeds will require new technology for these large masses. One proposal had been to use propulsive deceleration (PD) jets fired forward of the spacecraft ${ }^{1}$. Problems with such propulsive deceleration systems were identified to be flow stability, flow-control interaction and thermal protection of the aeroshell from the high-temperature PD jet gases. In order to design such systems, experimental data is needed to validate computational models and system studies. Most data dates from the 1960's and early 1970's and is summarized in Reference 2. When using propulsive deceleration it is desirable that the PD jet thrust not interact with the vehicle aerodynamics in such a way as to decrease the natural aerodynamic drag due to the vehicle bow shock. However, this early data established that a single PD jet on the centerline of the aeroshell forebody resulted in significant loss of aerodynamic drag, ie, little drag preservation. Some aerodynamic drag preservation was measured for PD jets positioned around the periphery of the aeroshell and at low thrust levels. A recent inviscid computational analysis predicted drag preservation using three and four PD jets placed near the aeroshell periphery, with some cases at angles to the aeroshell centerline ${ }^{3}$.

It is essential that experimental data be available to validate computational results of aerodynamic/propulsive interactions using PD jets. Experiments recently performed at NASA Langley produced flow visualizations of three peripheral PD jets ${ }^{4}$. The work reported herein uses a nonintrusive flow diagnostic, planar laser-induced iodine fluorescence, to collect in-stream data for single and peripheral PD jets in experiments at the University of Virginia. These data have been used to validate a computational code, LeMANS, developed at the University of Michigan. The validated code has been used to elucidate the flow physics associated with these propulsive deceleration systems. Some of these results are shown herein. The current research uses a single, central, PD jet and also 4 PD jets located mid way between the aeroshell centerline and shoulder. These jets are aligned with the aeroshell centerline, i.e., firing directly against the freestream. The freestream Mach number is 12. Comparisons with experimental data at Mach 6 show very good agreement.

\section{Experiment}

\section{A. Planar Laser-Induced Iodine Fluorescence (PLIIF) Measurement Technique}

PLIIF is a non-intrusive optical measurement technique which has been applied at the University of Virginia for quantitative measurements of all flow variables in continuum internal flows ${ }^{5-7}$ and continuum/rarefied external flows $^{8-10}$. This paper will focus on the application to measurements of PD jets in a Mach 12 freestream. The technique provides qualitative measurements of shock locations and jet concentration distributions and quantitative measurements of fluid density and jet mole fraction distributions. In such high-speed, low density flows, optical index-of-refraction-based techniques do not have enough sensitivity for imaging. Mechanical probes are intrusive and, as such, disturb the flowfield being investigated. Particle-based techniques, such as Particle Image Velocimetry, are not quantitative owing to particle slip across the strong shock waves in these low density flows. Other optical techniques, such as Mie scattering and nonlinear techniques, such as Raman scattering or Coherent Antistokes Raman Scattering, are ineffective owing to the low flowfield densities. It appears that only a laserinduced fluorescence approach will provide the measurement capability for this class of rarefied, hypersonic flows. Fluorescence from air or nitrogen flow would require complicated optical systems since they absorb only in the UV part of the spectrum. Iodine is unique since it is easy to seed into nitrogen or air flows in a room-temperature stagnation vessel and absorbs in the visible spectrum. It's absorption spectrum is accessible with standard argon ion lasers, operating at $514.5 \mathrm{~nm}$, and its emission is collected in the visible using standard CCD cameras having good visible response. Iodine is a solid at room temperature, with a 0.3 torr vapor pressure, but does not condense in these low-density flows since three-body collisions are needed to start the condensation process and these collision rates become vanishingly small in these rarefied flows. However, iodine fluorescence is used infrequently as a flow diagnostic since iodine is corrosive and somewhat toxic and requires special flow facilities and handling. With these precautions, however, it provides a unique and valuable measurement capability in hypersonic, low-density flows.

To understand the PLIIF technique requires examination of the iodine energy level diagram, Figure 1. The argon ion laser excites the iodine molecules from their rotational/vibration energy level in the ground, $\mathrm{X}$, state to an upper 
rotational/vibrational energy level in the $\mathrm{B}$ state. The molecules return to thermodynamic equilibrium via two processes, fluorescence and collisional quenching. These two processes compete and introduce the complication of the thermodynamics dependence of the collision rate in interpreting the fluorescence signal. These molecules then redistribute in other energy levels in the excited state and emit broadband fluorescence, which is shifted to longer wavelengths than that of the exciting laser. A rate-equation solution for the fluorescence, treating the process as two lumped energy levels, produces equation 1 for the fluorescence signal:

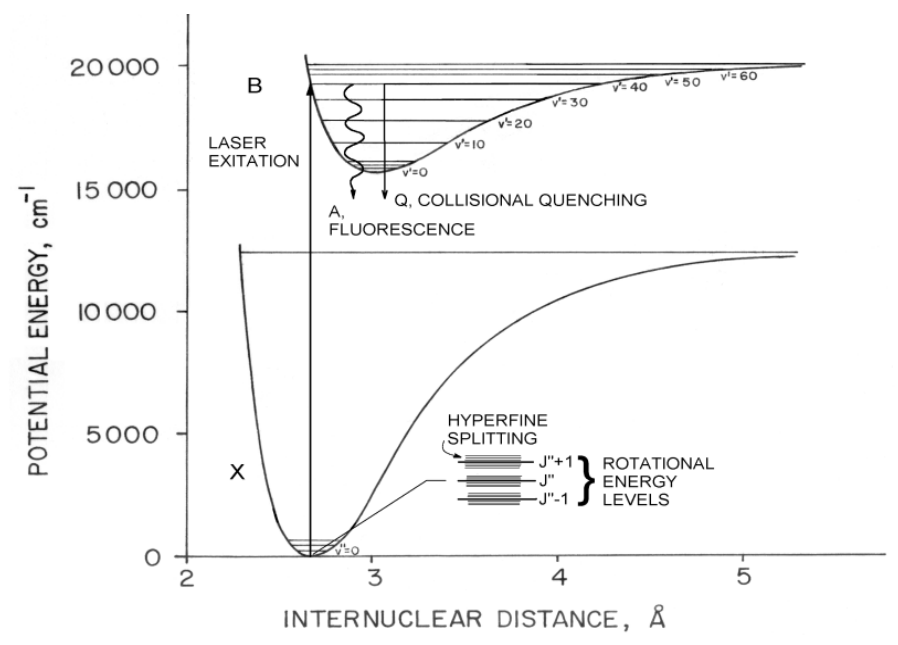

Figure 1: Energy level diagram of the iodine molecule showing fluorescence and collisional quenching rate processes.

$$
S_{F}=C f_{v^{\prime \prime} J^{\prime \prime}}(T) \frac{A_{21}}{A_{21}+Q(p, T)} \frac{\mathrm{V}(\mathrm{p}, \mathrm{T}, \mathrm{u})}{\Delta v_{D}(T)} I f_{S} n
$$

where the constant $\mathrm{C}$ contains molecular parameters and optical geometric factors and,

$f_{v^{\prime \prime} J^{\prime \prime}}(T) \quad$ is the Boltzmann population fraction of ground absorption level

$\mathrm{A}_{21} \quad$ is the fluorescence emission rate

$\frac{A_{21}}{A_{21}+Q(p, T)} \quad$ is the fluorescence efficiency (Stern-Volmer factor)

$Q(p, T) \quad$ is the follisional quenching rate

$\mathrm{V}(p, T, u) \quad$ is the line shape (Voigt function) of ground absorption transition (a function of pressure broadening, Doppler broadening and Doppler frequency shift)

$\Delta v_{D}(T) \quad$ is the Doppler line width

I is the laser intensity

$f_{s} \quad$ is the iodine seeding fraction

$n \quad$ is the number density.

The Boltzmann fraction is the product of the vibrational and rotational population fractions and depends on the vibrational and rotational quantum numbers, $\mathrm{v}$ " and $\mathrm{J}$ ". As will be seen, the values of these quantum numbers for the transitions at $514.5 \mathrm{~nm}$ enable measurements to be made in low temperature flows. The fluorescence efficiency 
factor is the ratio of the fluorescence decay rate to the total decay rate, fluorescence plus collisional quenching. The collisional quenching rate scales with the collisional frequency and therefore is a function of pressure, $\mathrm{p}$, and temperature, T. The lineshape of the absorbing transitions depends on pressure and Doppler broadening and is described by the Voigt function. The transitions are Doppler shifted when there is a velocity component, $\mathrm{u}$, of the molecules in the direction of the laser sheet. The Doppler line width depends on the temperature. I is the distribution of the laser intensity across the laser sheet. The seeding fraction is determined by the ratio of the room temperature vapor pressure of iodine to the flow stagnation pressure and, for the stagnation conditions of this work, is about 1 part iodine in 10,000 parts nitrogen. The last term is the total flowfield number density. It is seen that the expression for the fluorescence signal is a complex function of the flowfield conditions, which are not known a priori. In order to make a planar number density measurement, $p, T$ and $u$ must be known at all points in the plane defined by the laser sheet. This is not possible in general. In order to measure all these gas dynamic quantities, the laser is operated in the narrowband mode, using an intracavity etalon, such that the linewidth of the laser is much less than the linewidth of the iodine transition. This narrowband laser is tuned in frequency across the laser gain profile, and the iodine absorption spectrum is resolved. Then a spectral model for the iodine absorption spectrum, given by equation 1 , is fitted to the resolved spectrum and the gas dynamic parameters are determined. This has been the basis of previous studies ${ }^{5-10}$.

The alternate way to conduct a PLIIF measurement is to operate the argon laser in the broadband mode, with the intracavity etalon removed, such that the laser linewidth is much greater that the iodine absorption linewidth. In this case, the Voigt function, divided by the Doppler linewidth, integrates to unity since it is normalized. The fluorescence in this broadband mode is given by equation 2 .

$$
S_{F}=C f_{v^{\prime \prime} J^{\prime \prime}}(T) \frac{A_{21}}{A_{21}+Q(p, T)} I f_{s} n
$$

This approach has been used for quantitative planar temperature measurement ${ }^{11}$. This equation further simplifies in hypersonic, low temperature flows. The primary absorption transition under the argon laser gain profile has a low rotational quantum number of 14 (actually two overlapping lines with $\mathrm{J}=13$ and 15 ) and is in the ground, $\mathrm{v}=0$, vibrational level. Equation 3 gives the expression for the Boltzmann population fraction of the ground absorbing level:

$$
f_{J^{\prime \prime} v^{\prime \prime}}=f_{J^{\prime \prime}} \cdot f_{v^{\prime \prime}}
$$

where

$$
\begin{gathered}
f_{J^{\prime \prime}}=\frac{(2 J+1) \cdot \exp \left[-J(J+1) \frac{\theta_{\text {rot }}}{T}\right]}{\frac{1}{\sigma} \cdot \frac{T}{\theta_{\text {rot }}}} \\
f_{v^{\prime \prime}}=\exp \left(-v \frac{\theta_{v i b}}{T}\right) \cdot\left[1-\exp \left(-\frac{\theta_{v i b}}{T}\right)\right] \\
\theta_{\text {rot }}=0.0537 \mathrm{~K} \\
\theta_{v i b}=308.62 \mathrm{~K}
\end{gathered}
$$

In high Mach number flows from room stagnation temperature (the condition for the experiments discussed herein), the low temperatures produce a vibrational fraction of essentially unity and the rotational population collapses to low J". For iodine, the most populated rotational level at room temperature is about 57, but at the low freestream temperatures in these flows, about $10 \mathrm{~K}$, the most populated level is about 14. Hence the Boltzmann population fraction peaks at temperatures near the freesteam conditions. Figure 2 shows this Boltzmann population fraction as a function of Mach number in an isentropic expansion from room stagnation temperature. For the Mach 12 freestream conditions of the work reported herein, the fraction is near the maximum and is relatively constant from 
Mach 6-17. It is the low $\mathrm{J}$ " for the iodine absorption line accessible to the argon laser at $514.5 \mathrm{~nm}$ that makes this technique so powerful for low temperature flows.

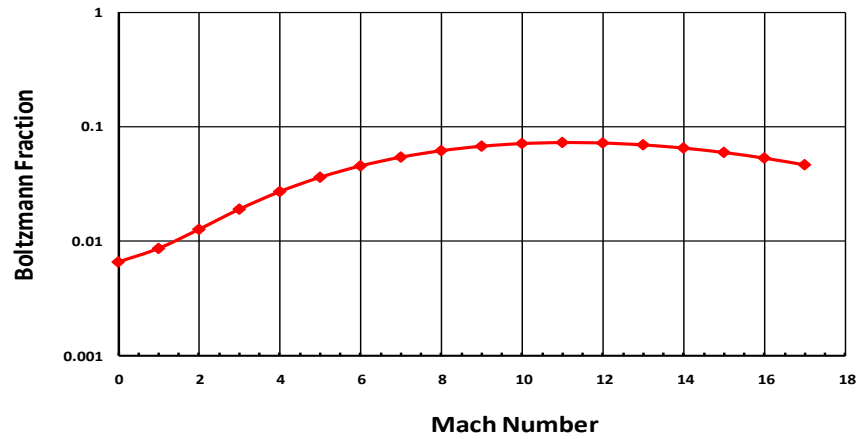

Figure 2: Boltzmann population fraction of iodine ground absorption transition versus Mach number in an isentropic expansion from room stagnation temperature.

The Stern-Volmer factor rapidly approaches unity in this isentropic expansion as the collisional decay rate approaches zero with decreasing $\mathrm{p}$ and $\mathrm{T}$. Figure 3 shows this factor versus Mach number. Therefore, between Mach numbers of about 6 and 17, the product of the Boltzmann fraction and the Stern-Volmer factor becomes nearly constant and the fluoresescence signal becomes directly proportional to the flowfield density. Figure 4 shows the broadband model for the fluorescence (equation 2) as the solid line. The blue points are experimental data in an isentropic expansion flowfield. Very good agreement is seen between the fluorescence model and the experimental data, validating the model given in equation 2. It is also seen that downstream of about 3 nozzle diameters, corresponding to Mach 6 in the underexpanded jet flowfield, the fluorescence signal decreases monotonically as does the density in this hypersonic, rarefied regime; however, closer to the nozzle exit, in the more continuum regime, the signal initially increases due to the increase in the Boltzmann fraction and Stern-Volmer factor (as seen in figures 2 and 3). In this flow region the fluorescence signal is not proportional to density.

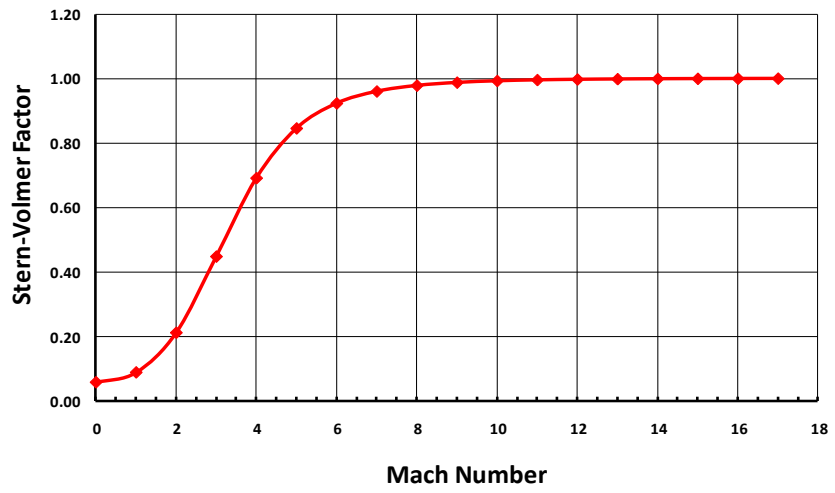

Figure 3: Stern-Volmer fraction versus Mach number in an isentropic expansion from room stagnation temperature.

Another quantitative measurement can be made when the experiment is conducted in the broadband laser mode. The approach is to take a PLIIF image with iodine seeded into the PD jet only. A second image is taken with the total flowfield (PD jet and freestream) seeded. When a ratio of the images is then taken, all the quantities that precede the number density in equation 2 , that are common in the numerator and denominator cancel. This is seen in equations 4. 


$$
\begin{gathered}
S_{F_{\text {Ratio }}}=\frac{C f_{v^{\prime \prime}, J^{\prime \prime}}(T) \frac{A_{21}}{C f_{v^{\prime \prime}, J^{\prime \prime}}(T) \frac{A_{21}}{A_{21}+Q(p, T)}} \frac{f_{S_{\text {Jet }}}}{f_{S_{\text {Total }}}} \frac{n_{\text {Jet }}}{n_{\text {Total }}}}{S_{F_{\text {Ratio }}}=A \frac{n_{\text {Jet }}}{n_{\text {Total }}}=A \chi_{\text {Jet }},}
\end{gathered}
$$

where the constant $\mathrm{A}$ is evaluated in the jet core where the mole fraction is unity. The fluorescence distribution in the ratioed image is proportional to the number density of the PD jet, divided by the total flowfield number density, or the PD jet mole fraction. The ratioed image therefore gives a quantitative measurement of the PD jet mole fraction distribution ${ }^{12-13}$. Note that this does not assume isentropic conditions and is, therefore, quantitative across shock waves and in viscous regions of the flowfield. This broadband mole fraction technique has been used to produce quantitative PLIIF images of the aerodynamic/propulsive interactions in PD jet flowfields.

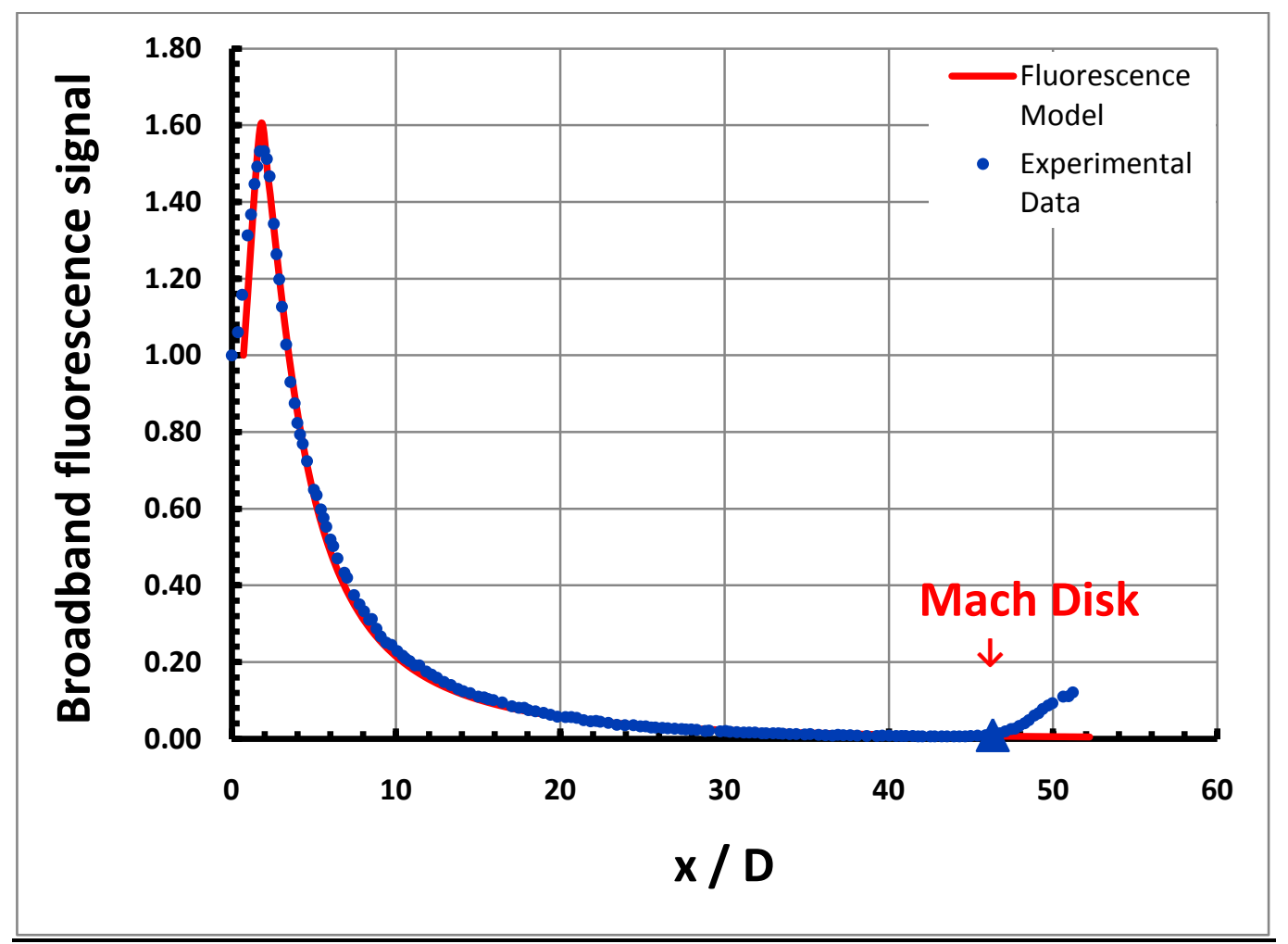

Figure 4: Fluorescence model (equation 2) and experimental data in isentropic expansion.

\section{B. Flow Facility}

The freestream flowfield is provided by an underexpanded jet. A large vacuum vessel is evacuated and the flow is expanded into the vacuum from a reservoir, producing the underexpanded jet. The freestream flowfield can be pure nitrogen, or nitrogen seeded with iodine. Figure 5 is a photograph of the vacuum vessel showing the large quartz windows which provide excellent optical access to the underexpanded-jet freestream flowfield. Optics for forming the PLIIF laser sheet are also seen in Figure 5. 


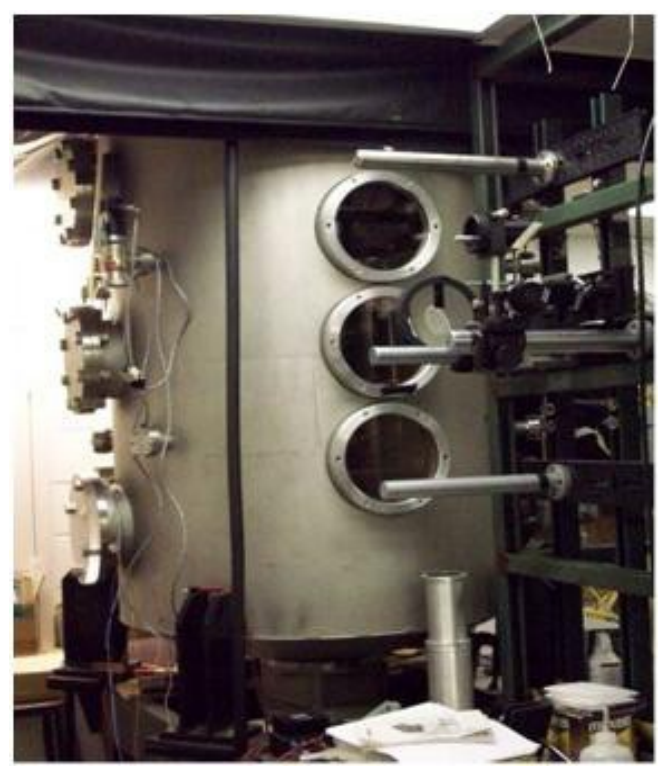

Figure 5: Vacuum vessel containing underexpanded-jet freestream flowfield.

The underexpanded jet is essentially a source flow which contains an isentropic core, surrounded by an oblique "barrel" shock and terminating in a normal shock, or "Mach disk." Figure 6 is a CFD calculation of the underexpanded-jet flowfield ${ }^{14}$. It can be seen that the flow expands from the nozzle exit at Mach 1 to values of about 17 before the Mach disk. The streamlines are divergent, as for a source flow, but this divergence is accounted for in the CFD calculations to be shown. The Knudsen number of the flow increases to about 1 just before the Mach disk, using the jet exit diameter as the characteristic length. The variation of the Mach number along the jet centerline is given by a relationship based on the Method of Characteristics ${ }^{15}$. The variation of the other flowfield parameters is given by the isentropic flow relations. The spacecraft model is placed in the underexpanded-jet freestream at a location to give the desired freestream Mach number. The model is shown to scale in Figure 6, placed about midway in the isentropic core where the Mach number is 12 . 


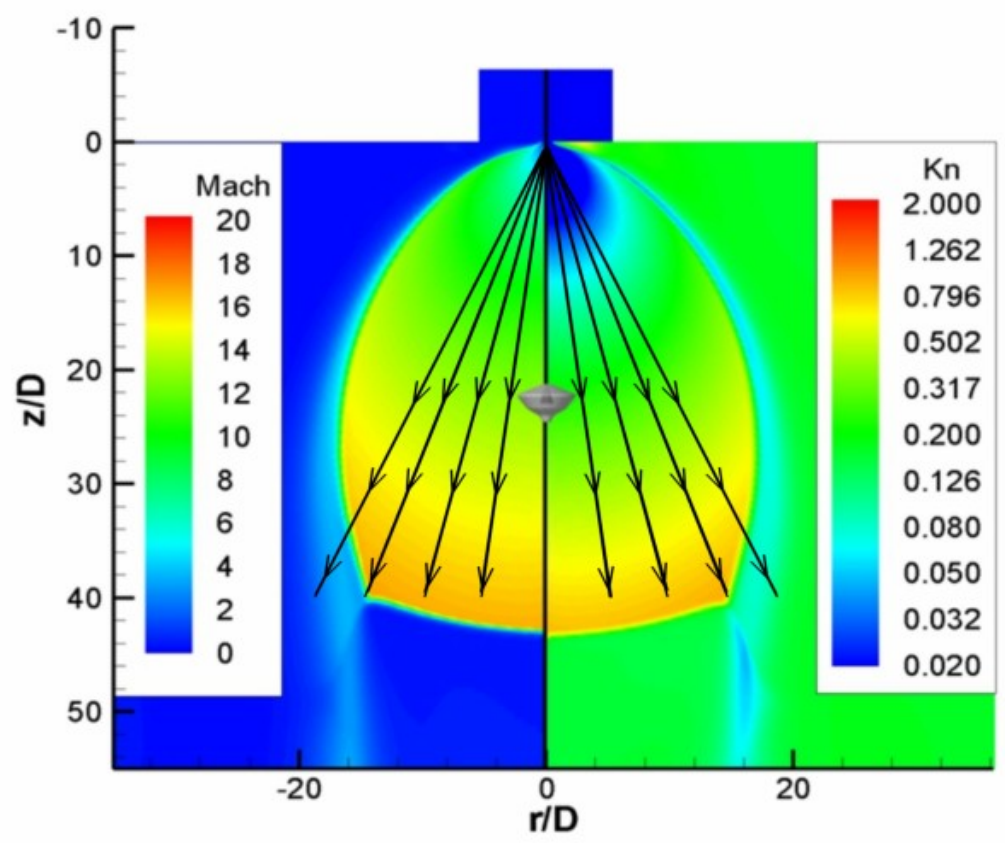

Figure 6: Variation of Mach and Knudsen numbers in experimental flowfield, showing source flow streamlines and the spacecraft model location.

\section{Optical Setup}

Figure 7 shows the optical setup for the PLIIF technique. An argon ion laser is operated on the $514.5 \mathrm{~nm}$ line. In the narrowband mode, the laser beam is split and a portion sent to a spectrum analyzer to monitor the laser frequency. A portion is also sent to an iodine static cell to provide an unshifted spectrum from which to measure the Doppler frequency shift. For the research reported herein, the laser is operated broadband and these components are not used. The laser beam is then converted into a thin laser sheet and directed into the vacuum chamber so as to impinge on the model on the plane to be measured. The planar fluorescence is collected by a cooled CCD camera, with exposure times from 15-30 seconds. An optical filter is placed in front of the camera to block the elastic scattering from the laser sheet.

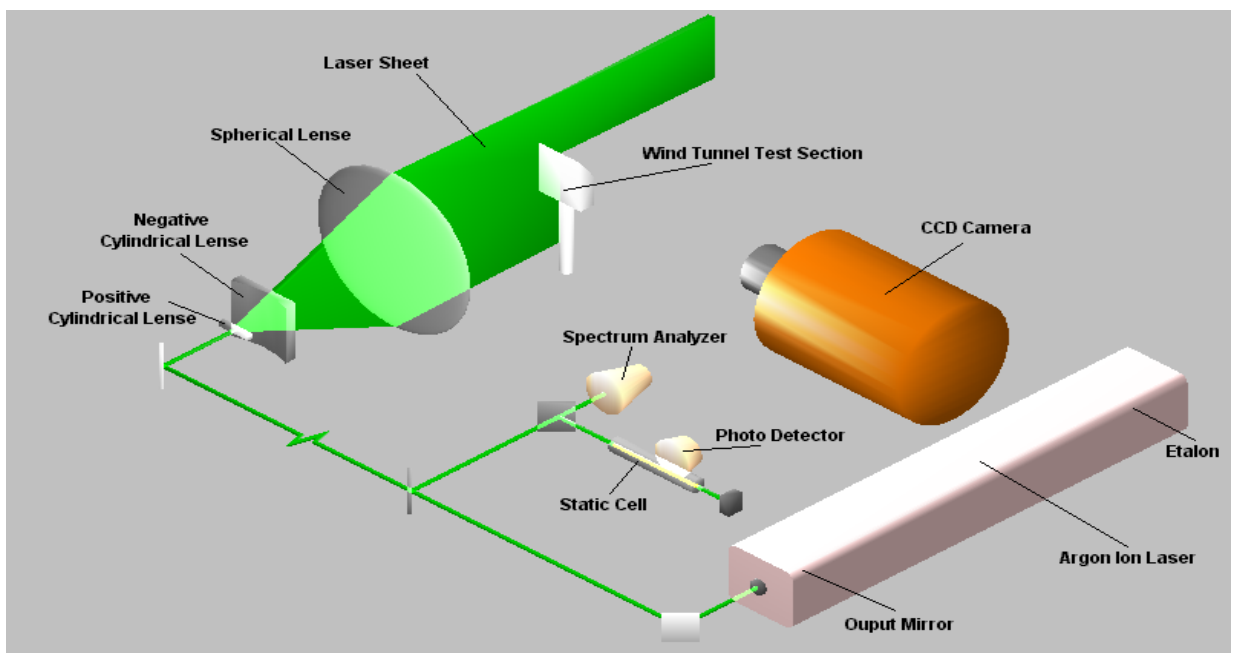

Figure 7: Optical setup for PLIIF technique.

8

American Institute of Aeronautics and Astronautics 


\section{Computational Fluid Dynamics}

LeMANS is a computational fluid dynamic (CFD) code developed at the University of Michigan for simulating hypersonic reacting flows ${ }^{16,17}$. LeMANS solves the laminar Navier-Stokes equations on unstructured computational grids including thermo-chemical nonequilibrium effects with second-order accuracy. In LeMANS, the translational and rotational energy modes of all species are described by two different temperatures $T_{t}$ and $T_{r},{ }^{18}$ respectively, while the vibrational energy mode and electron energy of all species are described by a single temperature $T_{v}$. In this study, Wilke's semi-empirical mixing ${ }^{19}$ is used with species viscosities calculated using Blottner's model ${ }^{20}$ and species thermal conductivities computed using Eucken's relation ${ }^{21}$. LeMANS uses the finite-volume method to solve the set of partial differentional equations. A modified Steger-Warming Flux Vector Splitting scheme is employed to discretize the inviscid fluxes across cell faces, while the viscous terms are computed using cell-centered and nodal values. Time integration is performed using either a point-implicit or a line-implicit method. LeMANS is parallelized using domain decomposition.

\section{Results}

PLIIF images and CFD calculations will be shown for single and peripheral PD jets over a range of thrust coefficients. The thrust coefficient, $\mathrm{C}_{\mathrm{T}}$, is defined as the thrust divided by the product of the freestream dynamic pressure and model frontal area, and is given by equation 5 :

$$
C_{T}=\frac{T}{q_{\infty} S}=\frac{\dot{m} u_{e}+\left(p_{e}-p_{\infty}\right) A_{e}}{q_{\infty} S}
$$

The first term is the product of the jet mass flow rate and exit velocity and the second term is the difference between the jet exit pressure and the freestream static pressure times the jet exit area. The freestream static pressure is taken as the pressure downstream of the bow shock wave.

\section{A. Central PD jet}

A CFD calculation of the flowfield resulting from a single, sonic centrally-located, PD jet issuing into a Mach 12 freestream with a thrust coefficient of 2.0 is shown in Figure 8 . The bow shock ahead of the jet flowfield compresses the freestream to subsonic speeds. The PD jet accelerates through its isentropic core and then compresses to subsonic flow across its Mach disk. On either side of the PD jet, recirculation regions are formed. A contact surface separates the freestream flow from the jet flow. This contact surface provides an "effective body shape" presented by the spacecraft model and PD jet flowfield. 


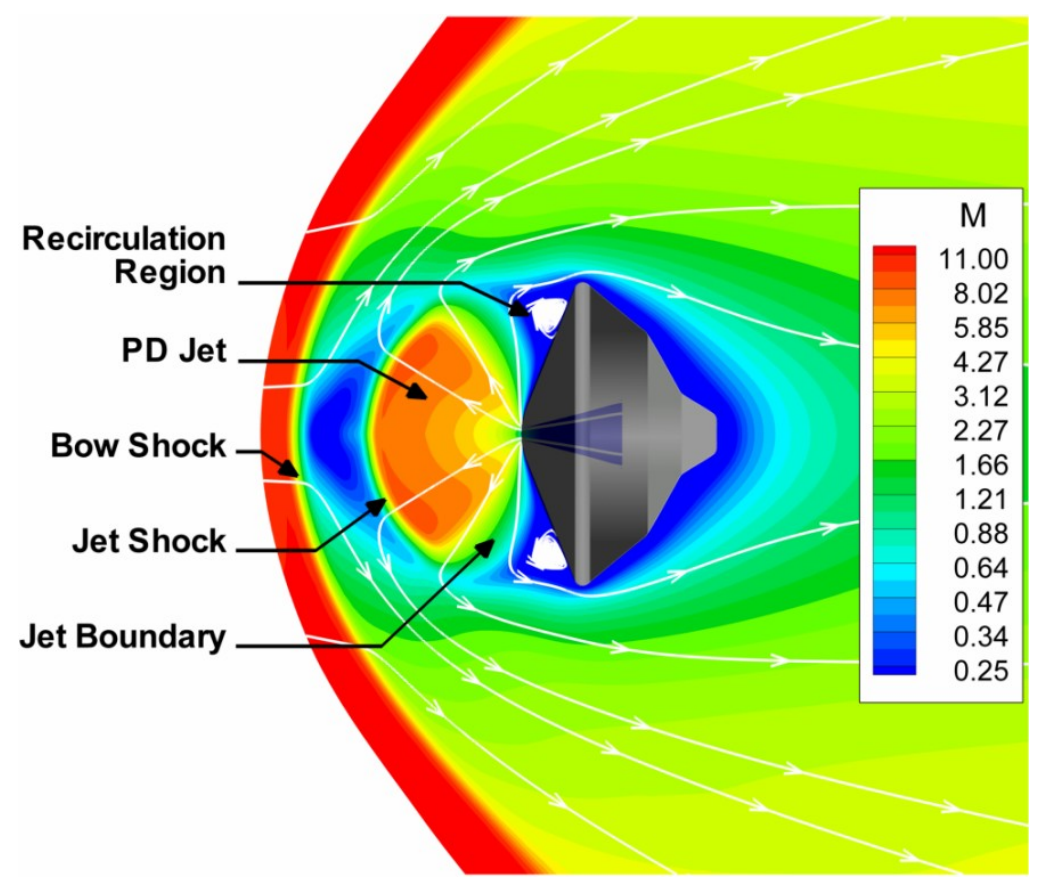

Figure 8. LeMANS calculation of Mach 12 flow over the spacecraft model with single sonic central PD jet at a thrust coefficient of 2.0 .

Figure 9 shows a series of PLIIF images of Mach 12 flow over the spacecraft model. The flow is from left to right in the images. The mounting sting for the model has been removed from the images. The freestream and PD jet flowfields are seeded with iodine in these images, so that the entire flowfield is visible. For these images the PD jet is sonic at the exit. The first image at $C_{T}=0$ shows the bow shock generated by the model with no PD jet. As the thrust coefficient is increased, this bow shock is pushed ahead of the model and the PD jet core increases in size. Note that for $\mathrm{C}_{\mathrm{T}}=1$ the bow shock becomes more oblique than the jet-off case, suggesting a drag decrease due to the weaker shock, acting like an aerospike; however, for higher $\mathrm{C}_{\mathrm{T}}$ the bow shock actually is more normal than the jet-off case. The change in bow shock shape is not the mechanism for the drag reduction with increasing thrust. 

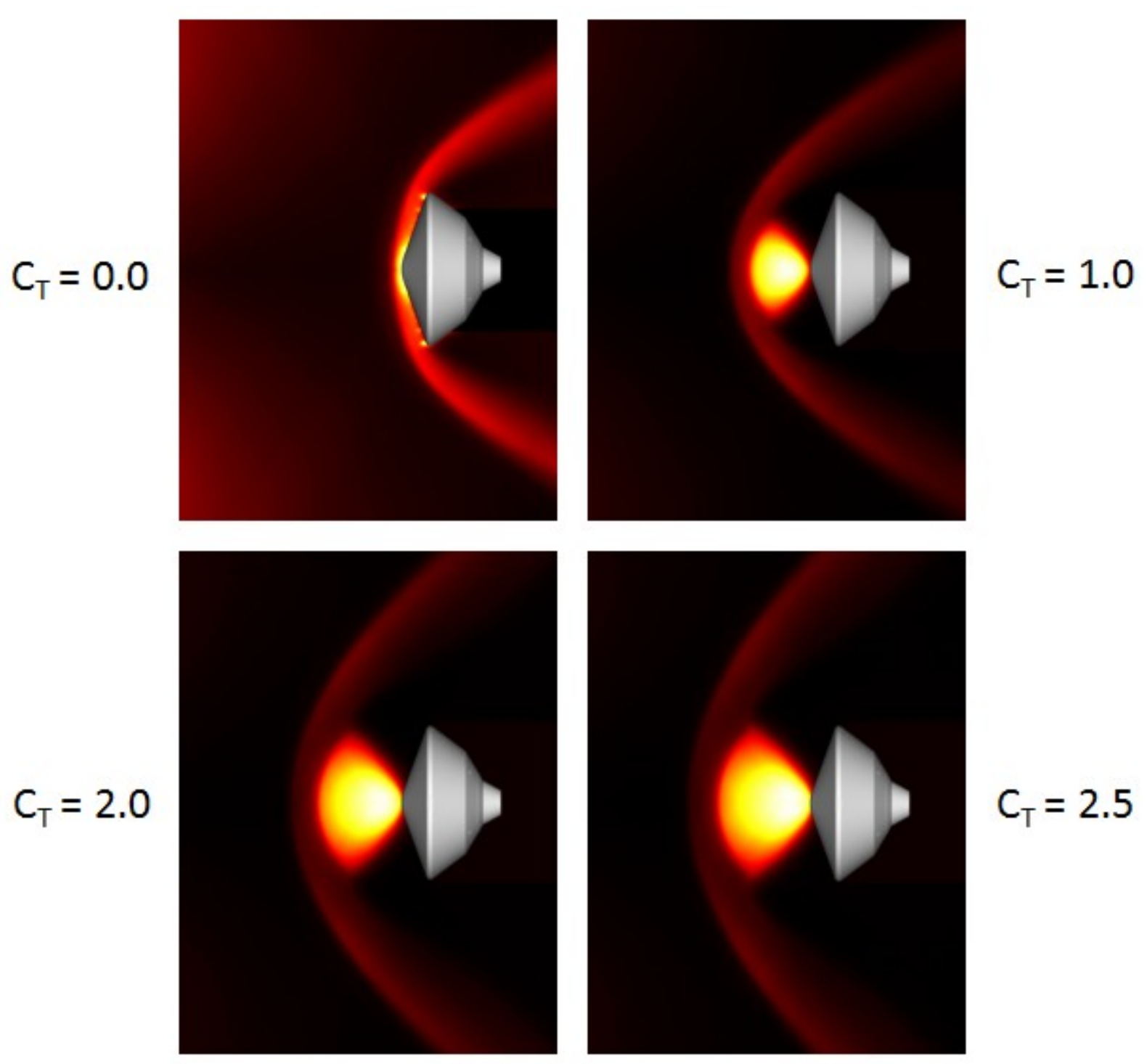

Figure 9. PLIIF images of spacecraft model with single sonic central PD jet for range of thrust coefficients.

References 22 and 23 show that the LeMANS calculations agree very well with the measured fluorescence images, both with respect to the streamline shapes and the shock standoff distance. These very good comparisons provide confidence that LeMANS is accurately capturing the details of the flowfield and can be used to calculate values, such as the drag coefficient, that cannot be measured in the experiment.

Figure 10 is a comparison of drag and total axial force coefficients from experiments by McGhee ${ }^{24}$ and calculations using LeMANS. It is seen that the drag coefficient decreases quickly with increasing thrust coefficient. This is because the jet lifts the bow shock off the aeroshell, decreasing the surface pressure relative to the jet-off case. The drag coefficient is about $1 / 6$ that of the jet-off case for a thrust coefficient of 2.0. The total axial force coefficient reaches a minimum at a thrust coefficient of about 0.5 and then increases as the thrust coefficient increases. The experimental and computational values agree well, again giving confidence in the LeMANS calculations. It is important to note that the McGhee data was taken at a freestream Mach number of 6 , whereas the LeMANS calculation was performed for Mach 12. This is a demonstration of hypersonic Mach number independence and indicates that the drag and axial force coefficients for the measurements reported herein are applicable to much lower Mach numbers. 


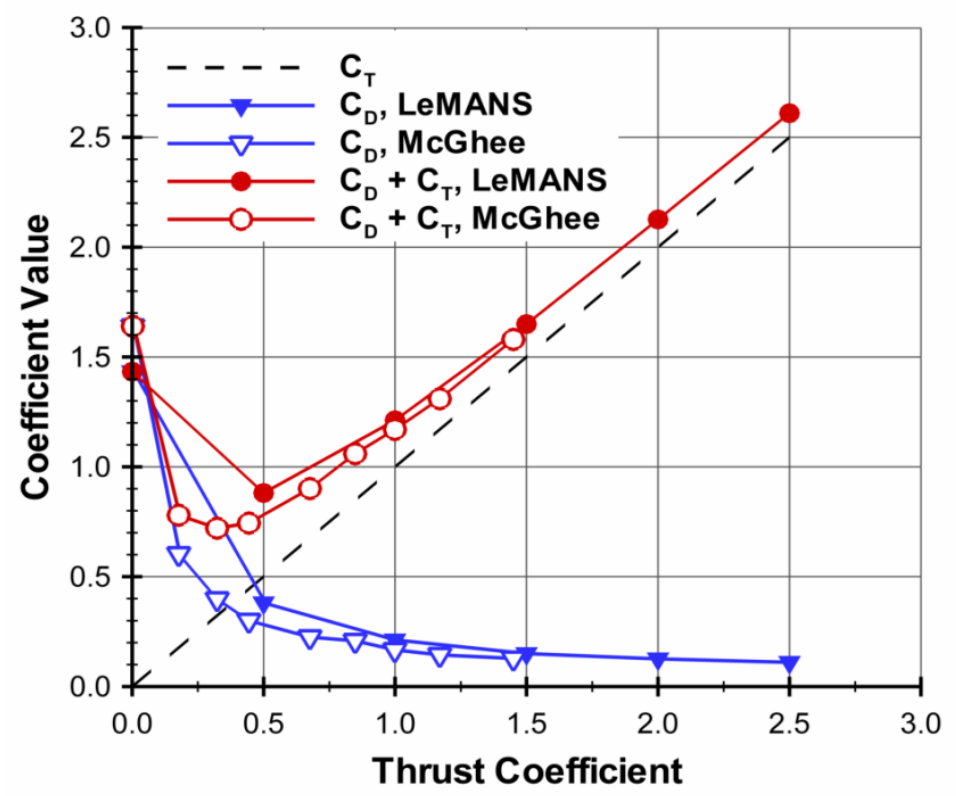

Figure 10. Comparison of experimental (McGhee) and CFD (LeMANS) drag and total axial force coefficients (from reference 25).

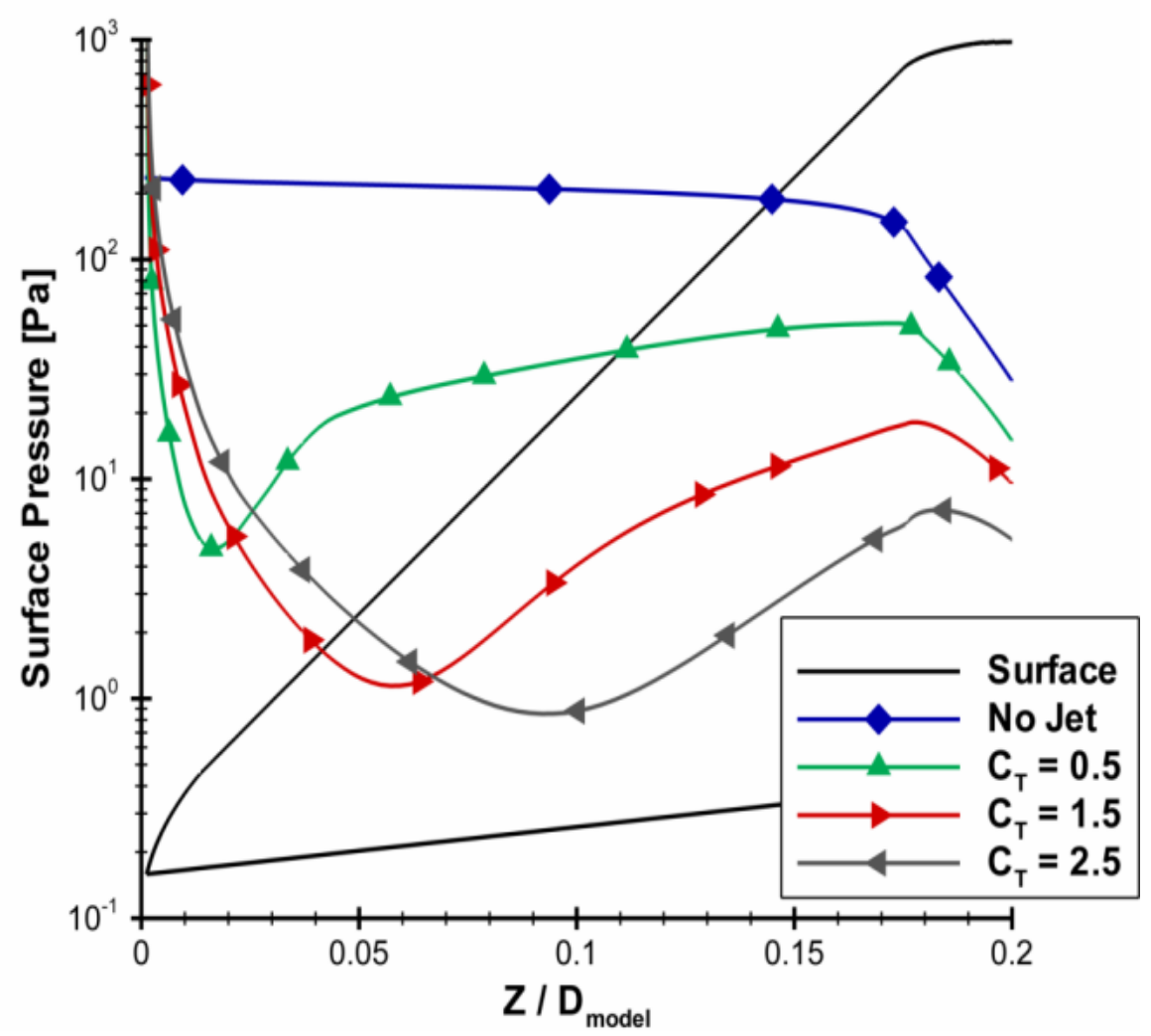

Figure 11. Surface pressure distributions for no jet and three values of the single PD jet thrust coefficient.

Figure 11 shows why the drag coefficient decreases with increasing thrust coefficient. It is seen that the surface pressure decreases by about a factor of 40 very close to the jet exit for $\mathrm{C}_{\mathrm{T}}=0.5$ and continues to decrease as the thrust coefficient is further increased. It is the lifting of the jet bow shock away from the aeroshell surface that 
causes surface pressure to decrease, decreasing the drag coefficient. Figure 12 shows this decrease in the surface pressure with increasing thrust coefficient. The left half of the figure is for the jet-off case, showing the high pressures behind the vehicle bow shock and the expansion from the center of the aeroshell to the shoulder. The much lower surface pressures are seen on the right with the PD jet on.

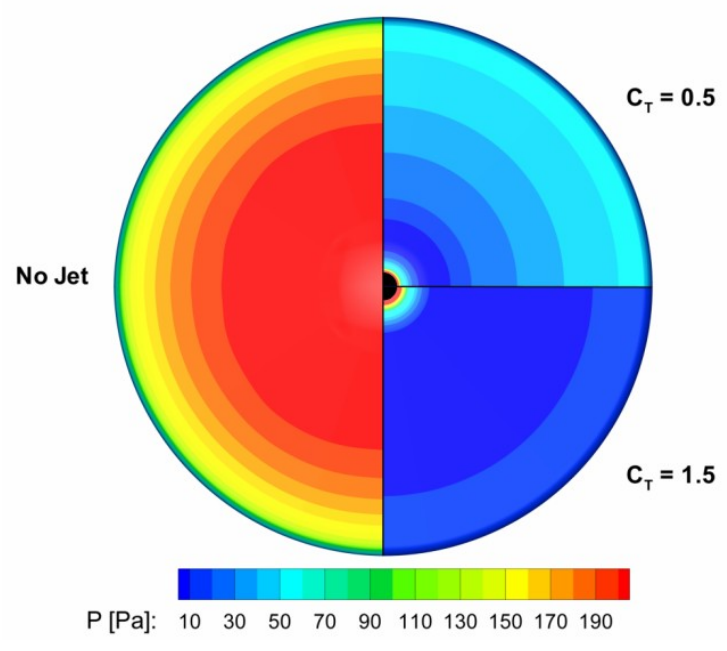

Figure 12. Surface pressure distribution for no jet and two single jet thrust coefficients.

\section{B. Peripheral 4-jet Model}

The structure of the flowfield for a model containing four sonic PD jets, located half way between the center of the aeroshell and the shoulder, is shown in Figure 13. The PD jets are again underexpanded and are swept to the side by the freestream flow. It is very important to note that a bow shock is maintained over about half of the model frontal area at this value of thrust coefficient. It will be shown that the presence of this bow shock preserves significant aerodynamic drag relative to the single centerline PD jet. It should also be noted that the outermost streamlines locate the contact surface between the jets and the freestream.

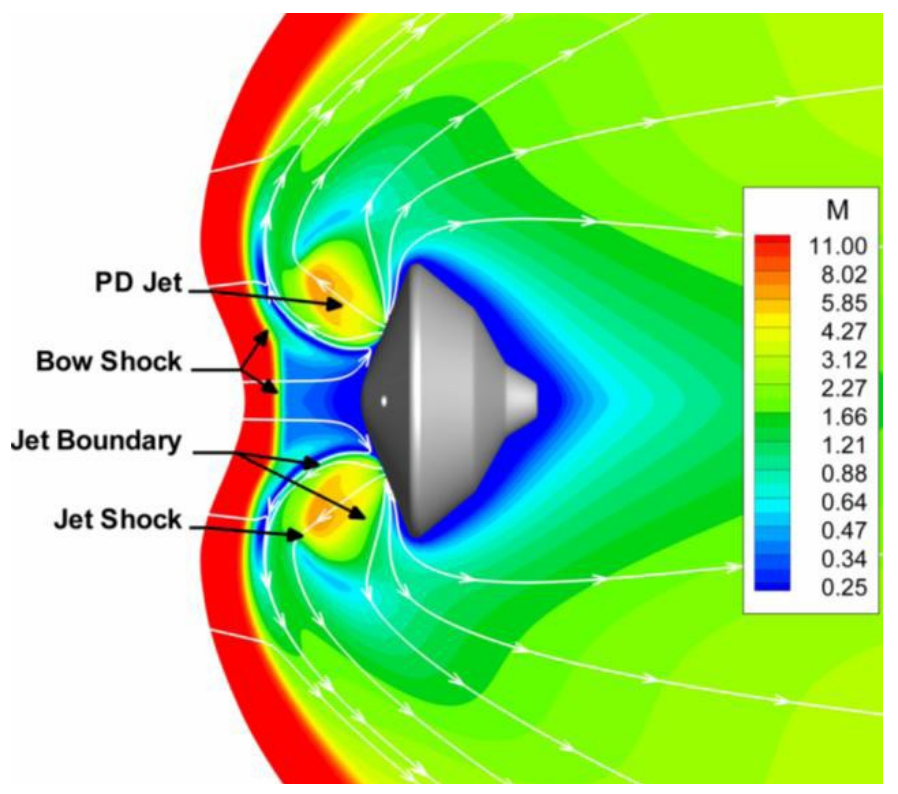

Figure 13. CFD calculation of peripheral sonic PD jets for $C_{T}=1.5$.

American Institute of Aeronautics and Astronautics 
Figure 14 contains a series of PLIIF images of a cross-section of the peripheral jet flowfield, with the laser sheet intersecting the flowfield along the centerline of two of the jets. Again, the flow is from left to right with a freestream Mach number of 12. The PD jets are sonic at the exit. There are two more jets that are not seen since they are not in the plane of the laser sheet and therefore are not fluorescing. The important observation is that the bow shock is preserved between the jets and is pushed away from the aeroshell as the thrust coefficient is increased. It will be seen that the fluid between the PD jets is primarily from the compressed freestream and not jet fluid. For sonic PD jets with thrust coefficients greater than 1, the hot PD jet gases would not impinge on the vehicle surface, which is an important result for thermal protection system design.

Figure 15 is the results of a LeMANS calculation of the drag coefficient and total axial force coefficient versus thrust coefficient for a single central, jet and 4 peripheral jets. It is seen that, for the peripheral PD case, the drag coefficient is about $2 / 3$ of the jet-off drag coefficient for a thrust coefficient of 2.0. This should be compared to the single PD jet case where the drag coefficient is about $1 / 6$ of the jet-off drag coefficient at a thrust coefficient of 2.0. Therefore, the peripheral jet drag coefficient is four times that of the single jet at a thrust coefficient of 2.0. The total axial force coefficient increases monotonically for the peripheral case, with the drag coefficient being about $1 / 3$ of the total axial force coefficient at a thrust coefficient of 2.0. Clearly, the peripheral jets preserve the aerodynamic drag much more than the single central PD jet.

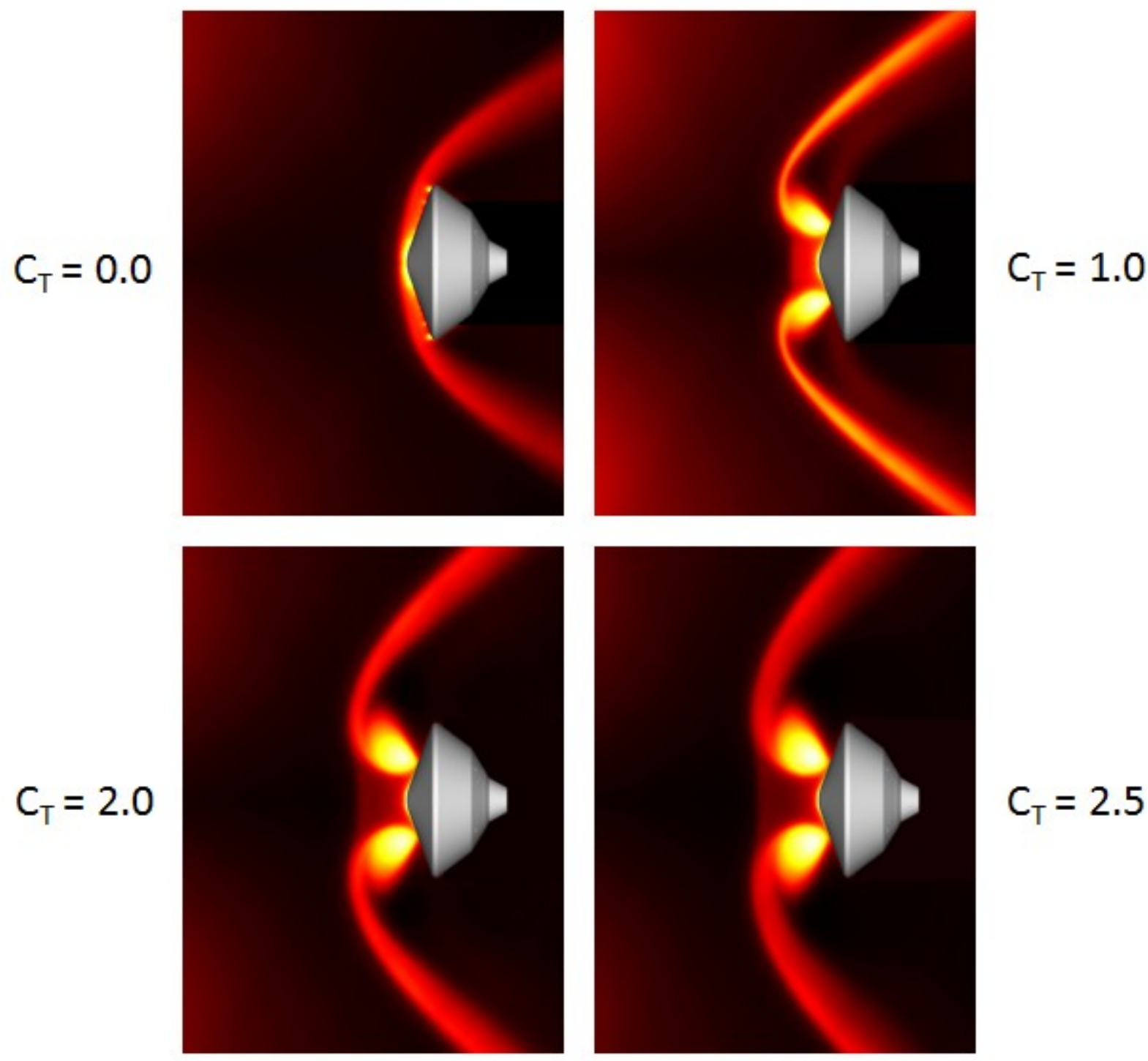

Figure 14. PLIIF images of peripheral sonic PD jets for increasing thrust coefficient. 


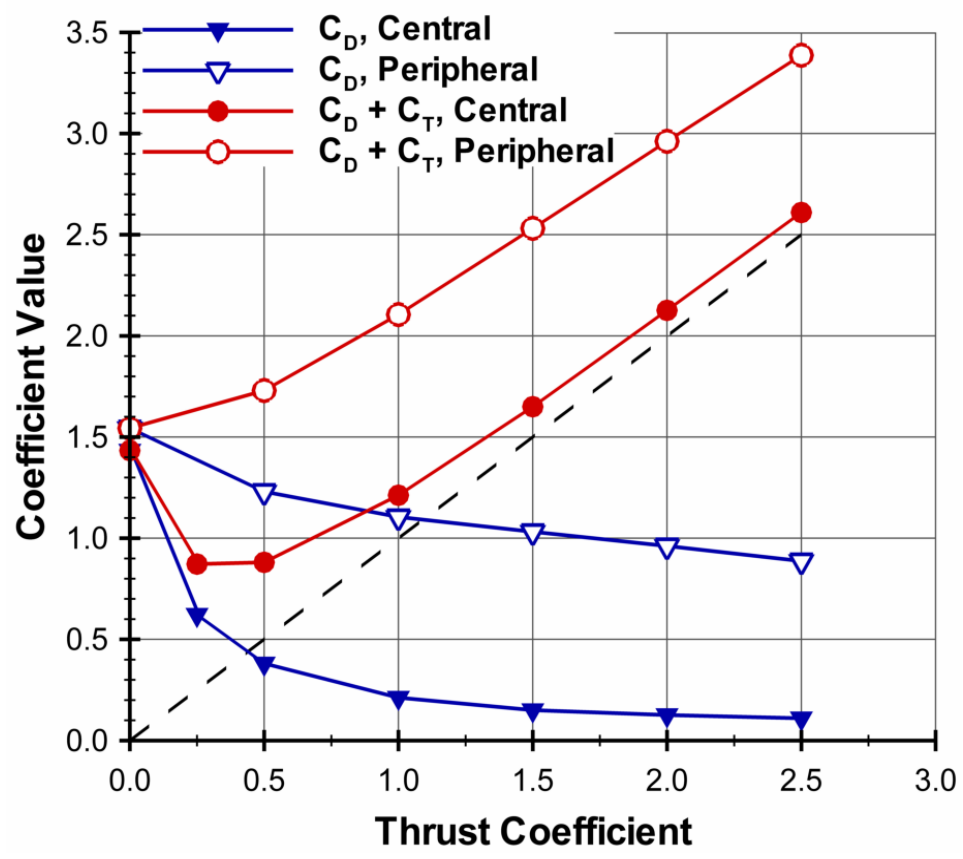

Figure 15. Drag and total axial force coefficients for single central and peripheral PD jets versus thrust coefficient (from reference 25).

The reason for the decrease in drag coefficient with increasing thrust coefficient is again seen from the surface pressure distribution. Figure 16 is a Mach number distribution taken just outside the boundary layer for one quadrant of the peripheral 4-jet case. It is seen that the flow is radial without the PD jet and with jets on it flows around the jet, as the jet appears as an obstacle to the radial flow. The streamlines join again in the wake of the jet. As the thrust coefficient increases, the jet provides a larger obstruction to the radial flow and the low Mach number wake increases behind the jet. Figure 17 shows that the wakes are regions of low pressure and increase in size as the thrust coefficient increases. This is the mechanism for the decrease in drag coefficient of the peripheral jets with increasing thrust coefficient, i.e. the low pressure wake becomes larger. This suggests that moving the jets closer to the shoulder would produce more drag preservation since the low pressure region would be moved off the surface. This is consistent with the calculations given in Reference 3 which showed greater drag preservation with PD jets located $85 \%$ of the distance from the aeroshell centerline to shoulder.

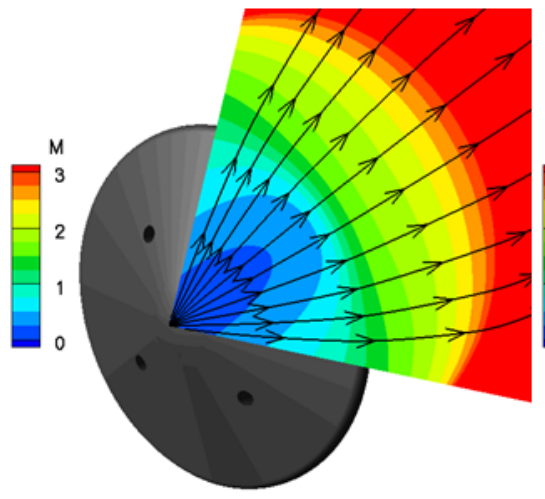

No Jet

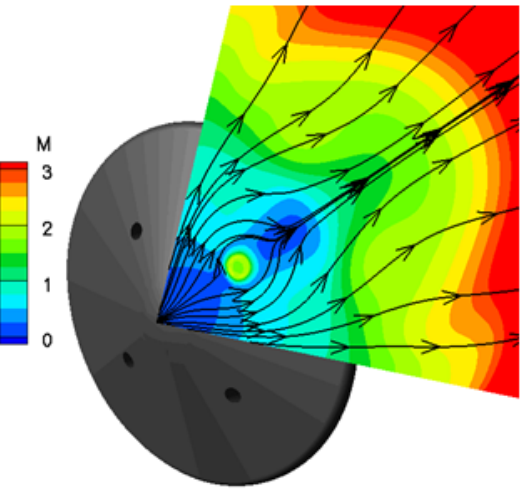

$\mathrm{C}_{\mathrm{T}}=0.5$

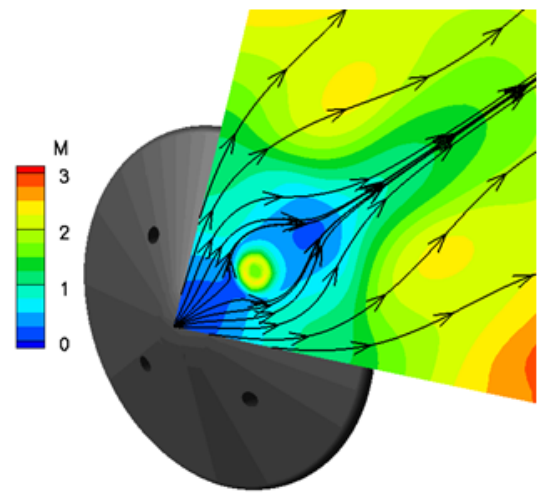

$\mathrm{C}_{\mathrm{T}}=1.5$

Figure 16. Mach number distribution just outside the boundary layer shown for one quadrant of the 4-jet model. 
Profiles of surface pressure distributions for the peripheral jets are shown in Figure 18. For the plane through the jets (left), the pressure between the center of the aeroshell and the jet is maintained at the level of the jet-off case. Maintaining this bow shock, as seen on the flowfield schematic and the PLIIF images, is responsible for the drag preservation. The pressure between the jet and shoulder is decreased due to the low pressure wake seen in Figure 17. It is seen that for the plane taken midway between the jets (right), the pressure distribution is maintained at the jet-off level since the bow shock is undisturbed between the jets. These distributions suggest that a few PD peripheral jets would preserve more of the drag coefficient since the region between the jets, where the surface pressure is the same as that for the jet-off case, would be larger.

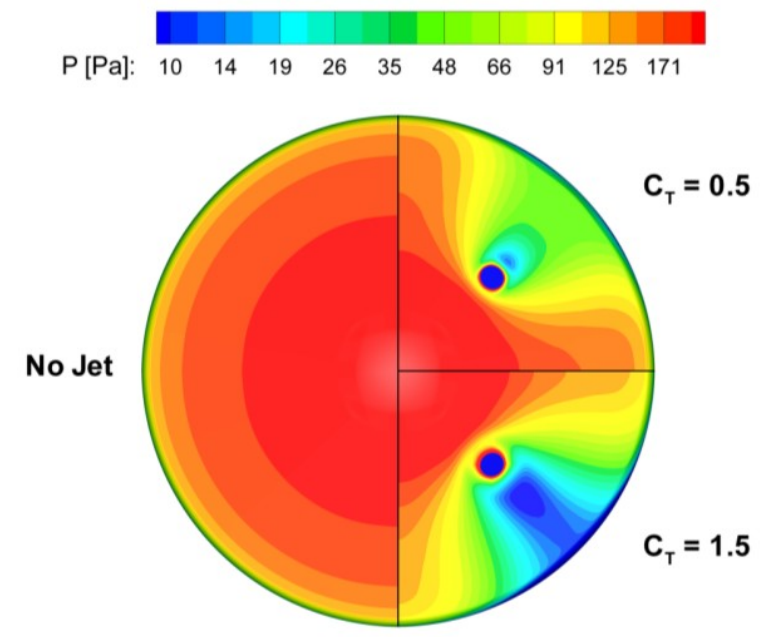

Figure 17. Surface pressure distribution for peripheral jets versus thrust coefficient.
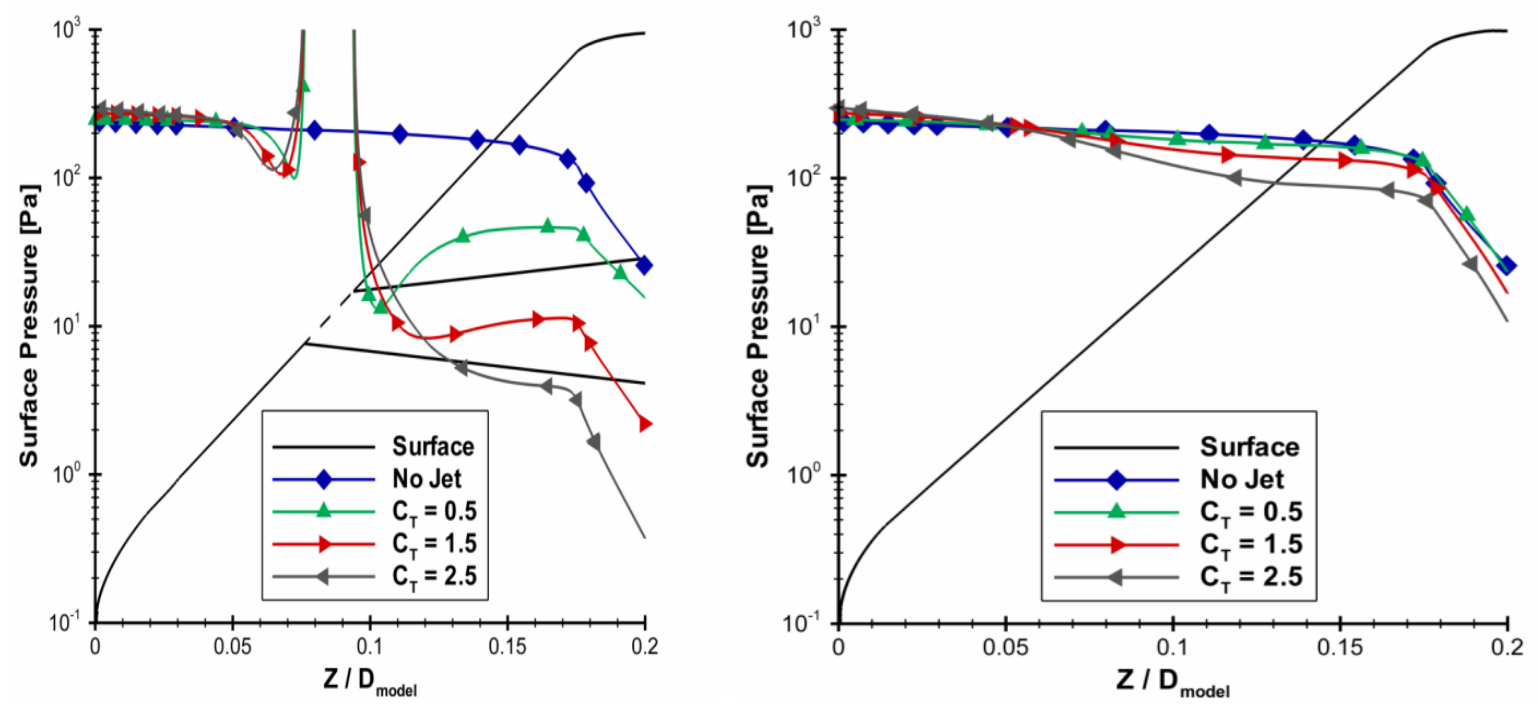

Figure 18. Surface pressure distributions on a plane through the peripheral jets (left) and between two jets (right).

Figure 19 is the mole fraction distribution for Mach 2.66 peripheral jets at a thrust coefficient of 1.5. The contours in this figure provide a quantitative planar distribution of the PD jet fluid. The jet mole fraction decreases from unity in the PD jet cores (dark red regions in the figure) to zero as the jets mix with the hypersonic freestream (dark blue regions). This image also shows that the region of the flowfield between the PD jets is not jet fluid, but compressed freestream fluid, as mentioned previously. 


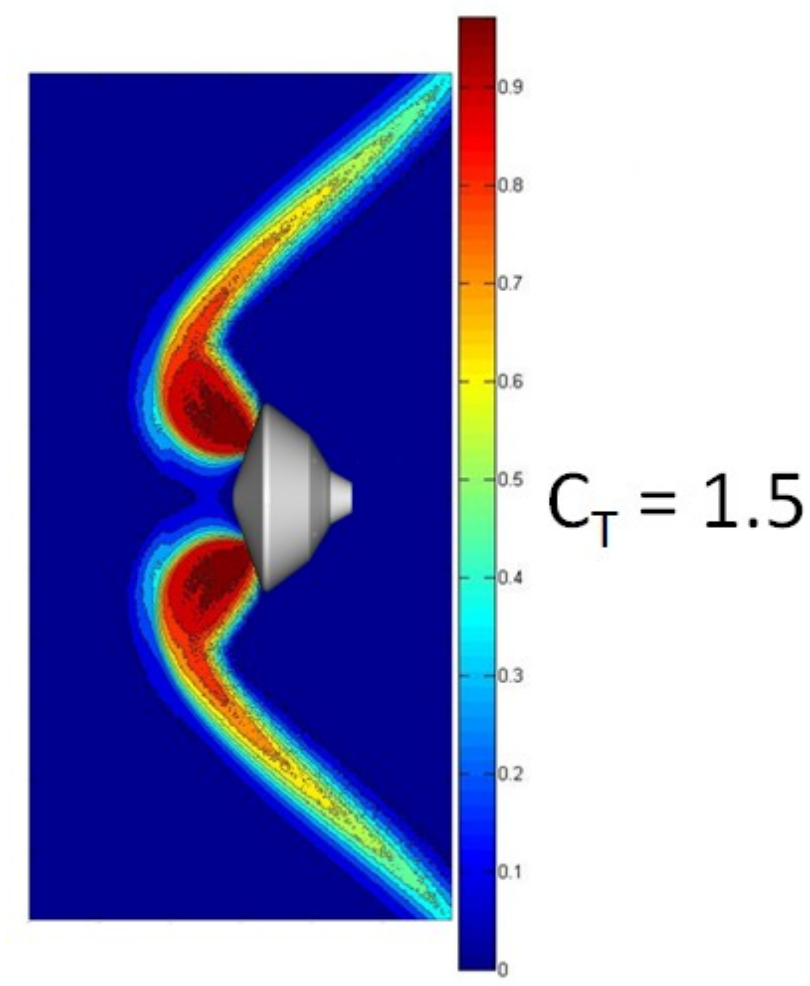

Figure 19. Jet mole fraction distribution for 4 sonic peripheral jets at a thrust coefficient of 1.5 .

A three-dimensional depiction of the sonic peripheral PD jets is given in Figure 20. The fluorescence from two intersecting laser sheets is shown. The figure on the left shows the model bow shock without jet, and the figure on the right is for the case of $\mathrm{C}_{\mathrm{T}}=3.0$ (as seen in Figure 14, lower right). The bright spots on the shoulder of the model for the figure on the right are scattering from the laser sheet. These images illustrate the capability of the PLIIF technique to image the complex flowfield associated with PD jets in a rarefied, hypersonic flow.

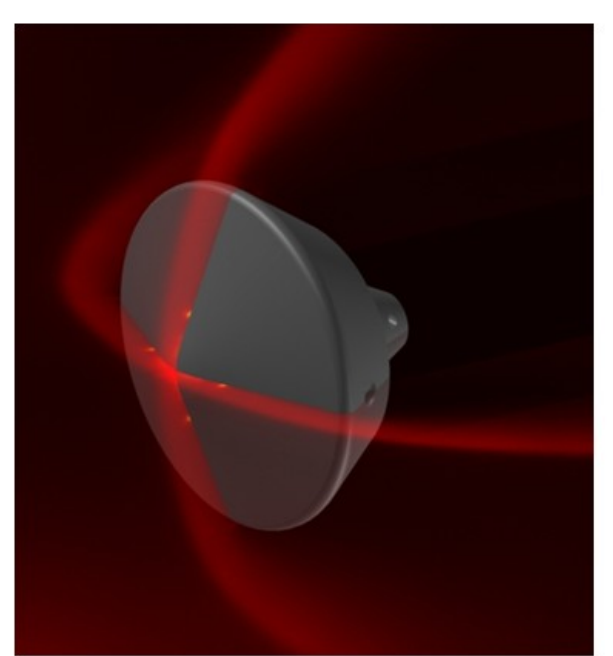

$$
\mathrm{C}_{\mathrm{T}}=0
$$

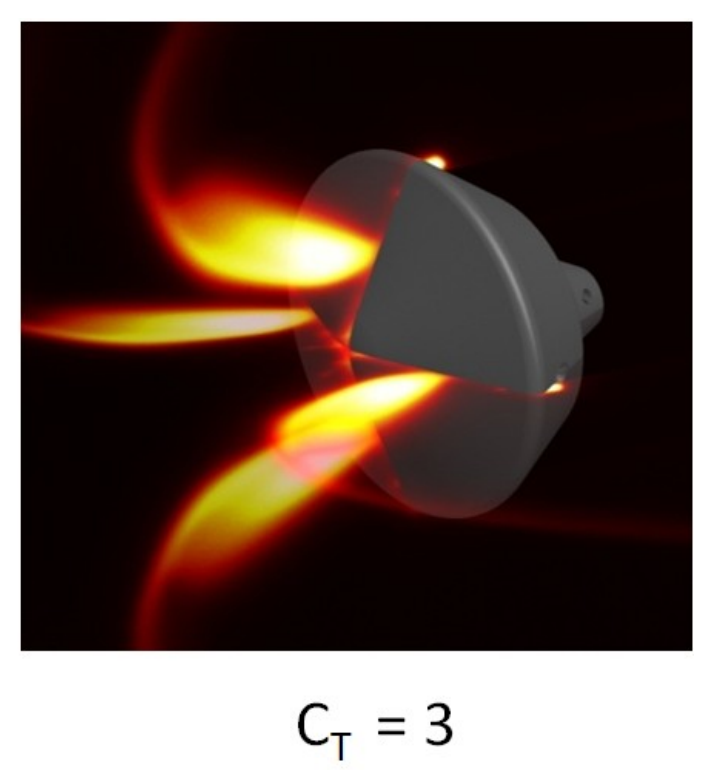

Figure 20. Three dimensional depiction of model bow shock (left) and peripheral PD jets (right)

American Institute of Aeronautics and Astronautics 


\section{Conclusion}

Propulsive deceleration (PD) jets have been investigated, both experimentally using PLIIF and computationally using a CFD code (LeMANS). The theory behind PLIIF was discussed. It was shown that the low rotational quantum number of the iodine molecule provides a unique capability for measurement in low temperature flows. PLIIF images were shown for a single central PD jet in Mach 12 flow, which showed lifting of the vehicle bow shock with increasing thrust coefficient. LeMANS calculations for the single jet showed a monotonically decreasing drag coefficient, to about $1 / 6$ of the jet-off case at a thrust coefficient of 2.0. The total axial force coefficient decreases to a minimum at a thrust coefficient of 0.5 and then increases with increasing thrust coefficient. This behavior was in good agreement with previous experimental data at Mach 6, illustrating hypersonic Mach number independence.

PLIIF images were also shown for a 4-jet peripheral model which showed preservation of the vehicle bow shock between the jets as the thrust coefficient increased. LeMANS calculations showed that with peripheral jets, a drag coefficient of about 2/3 of the jet-off case was achieved, 4 times that for the single PD jet. Calculations showed that the radial flow on the aeroshell surface produces low pressure wakes behind the peripheral jets, which are responsible for the decrease in drag coefficient with increasing thrust coefficient. This suggests that moving the peripheral jets closer to the shoulder would move the low pressure wakes off the aeroshell, preserving more of the drag coefficient. Also, between the peripheral jets the surface pressure was maintained at the jet-off level, suggesting that using a few peripheral jets, maintaining that high pressure between the jets, would preserve more of the vehicle drag coefficient. A PLIIF mole fraction image provides a measurement of the quantitative distribution of PD jet fluid and showed that the region on the aeroshell between the PD jets contained primarily compressed freestream fluid.

Future research will carried out by operating the PLIIF in the narrowband mode so that the molecular Doppler shift can be measured, providing a quantitative measurement of velocity throughout the PD jet flowfields. This data will be used for further detailed comparison with the LeMANS calculations.

\section{Acknowledgments}

This research was supported by NASA grant NNX08AH37A, funded as a NRA by NASA Aerospace Research Mission Directorate. Computation support of the University of Michigan Center for Advanced Computing is essential to this work and is greatly appreciated. The assistance of Dr. Paul Danehy and Scott Ashcraft, NASA Langley Research Center, in preparation of the images shown in figures 9, 14, 19 and 20 is most appreciated.

\section{References}

1. Braun, R.D. and Manning, R.M., "Mars Exploration Entry, Descent and Landing Challenges," Journal of Spacecraft and Rockets, Vol. 44, No. 2, 2007, pp. 310-323.

2. Korzun, A.M., Braun, R.D. and Cruz, J.R., "Survey of Supersonic Retropropulsion Technology for Mars Entry, Descent and Landing," Journal of Spacecraft and Rockets, Vol. 46, No. 5, 2009, pp. 929-937.

3. Bakhtian, N.M. and Aftosmis, M.J., "Parametric Study of Peripheral Nozzle Configurations for Supersonic Retropropulsion,” Journal of Spacecraft and Rockets, Vol. 47, No. 6, 2010, pp. 935-950.

4. Palaszewski, B. and Bencic, T., "Mars Entry, Descent and Landing: Experiments with Multiple Engine Supersonic Retro Propulsion Configuration," AIAA 2010-6594, Joint Propulsion Conference, Nashville, TN, July 2010.

5. Fletcher, D.G. and McDaniel, J.C., "Laser-Induced Iodine Fluorescence Technique for Quantitative Measurement in a Nonreacting Supersonic Combustor," AIAA Journal, Vol. 27, No. 5, May 1989, pp. 575-580.

6. Hartfield, R.J., Hollo, S.D. and McDaniel, J.C., "Experimental Investigation of a Supersonic Swept Ramp Injector using Laser-Induced Iodine Fluorescence," Journal of Propulsion and Power, Vol. 10, No. 1., Jan.-Feb. 1994, pp. 129-135. 
7. Donohue, J.M. and McDaniel, J.C., "Complete 3-D Multi-Parameter Mapping of a Swept Ramp Fuel Injector using Planar Laser-Induced Iodine Fluorescence,” AIAA 1995-0423, Aerospace Sciences Meeting, Reno, NV, Jan. 1995

8. Cecil, D.E. and McDaniel, J.C., "Planar Laser-Induced Iodine Fluorescence Measurements in Rarefied Hypersonic Flow," International Rarefied Gas Dynamics Symposium, Toronto, Canada, 2005, pp. 1325-1350.

9. McDaniel, J.C., Cecil, D.E., Reed, E.M. and Codoni, J.R., "Planar Laser-Induced Iodine Fluorescence Technique for Flow Visualization and Quantitative Measurements in Rarefied Flows," International Rarefied Gas Dynamics Symposium, Monterey, CA, July 2010.

10. Cecil, D.E. and McDaniel, J.C., "Direct Monte Carlo Simulations of a Binary Gas Free-Jet Flow over a Flat Plate," International Rarefied Gas Dynamics Symposium, Monterey, CA, July 2010.

11. Hartfield, R.J., Hollo, S.D. and McDaniel, J.C., "Planar Temperature Measurement in Compressible Flows using Laser-Induced Iodine Fluorescence," Optics Letters, Vol. 16, Jan. 1991, pp. 106-108.

12. Hartfield, R.J., Abbitt, J.D. and McDaniel, J.C., "Injectant Mole Fraction Imaging in Compressible Mixing Flows using Planar Laser-Induced Iodine Fluorescence," Optics Letters, Vol. 14, August 1989, pp. 850-852.

13. Abbitt, J.D., Hartfield, R.J. and McDaniel, J.C., "Mole Fraction Imaging of Transverse Injection in a Ducted Supersonic Flow," AIAA Journal, Vol. 29, No. 3, March 1991, pp. 431-435.

14. McDaniel, J.C., Glass, C.E., Staack, D. and Miller, C.G., "Experimental and Computational Comparisons of an Underexpanded Jet Flowfield," AIAA 2002-0305, Aerospace Sciences Meeting, Reno, NV, January 2002.

15. Ashkenas, H. and Sherman, F.S., "The Structure and Utilization of Supersonic Free Jets in Low Density Wind Tunnels," Rarefied Gas Dynamics, Vol. 2, Academic Press, New York, 1966.

16. Scalabrin, L. C. and Boyd, I. D., "Development of an Unstructured Navier-Stokes Solver for Hypersonic Nonequilibrium Aerothermodynamics," AIAA 2005-5203, June 2005.

17. Scalabrin, L.C. and Boyd, I.D., "Numerical Simulation of Weakly Ionized Hypersonic Flow for Reentry Configurations," AIAA 2006-3773, June 2006.

18. Holman, T.D. and Boyd, I.D., "Effects of Continuum Breakdown on the Surface Properties of a Hypersonic Sphere," Journal of Thermophysics and Heat Transfer, Vol. 23, No. 4, Oct. - Dec. 2009, pp. 660-673.

19. Wilke, C.R., "A Viscosity Equation for Gas Mixtures," Journal of Chemical Physics, Vol. 18, No. 4, 1950, pp. 517-519.

20. Blottner, F.G., Johnson, M., and Ellis, M., "Chemically Reacting Viscous Flow Program for Multi-Component Gas Mixtures,” Tech.rep.,SC-RR-70-754, Sandia Laboratories, Albuquerque, NM, 1971.

21. Vincenti, W.G. and Kruger, C.H., Introduction to Physical Gas Dynamics, Krieger Publishing Company, 2002.

22. Alkandry, H, Boyd, I.D., Reed, E.M., Codoni, J.R. and McDaniel, J.C., "Interactions of Single-Nozzle Sonic Propulsive Deceleration Jets on Mars Entry Aeroshells," AIAA 2010-4888, June 2010

23. Alkandry, H, Boyd, I.D., Reed, E.M., Codoni, J.R. and McDaniel, J.C., "Interactions of Single-Nozzle Supersonic Propulsive Deceleration Jets on Mars Entry Aeroshells, AIAA 2011-138, Aerospace Sciences Meeting, Jan. 2011

24. McGee, R.J., "Effects of a Retronozzle Located at the Apex of a 140 degree Blunt Cone at Mach Numbers of 3.00, 4.50 and 6.00," NASA Technical Note D-6002, Jan. 1971.

25. Codoni, J.C., Reed, E.M., McDaniel, J.C., Alkandry, H. and Boyd, I.D., "Investigations of Peripheral 4-Jet Sonic and Supersonic Propulsive Deceleration Jets on a Mars Science Laboratory Aeroshell, AIAA 2011-1036, Aerospace Sciences Meeting, Jan. 2011 